\title{
Otoesclerosis. Aspectos histopatológicos y resultados auditivos de la estapedostomía
}

\section{Otosclerosis. Histopathological features and stapedostomy hearing results}

\author{
Carlos Stott C' , Alejandro 0jeda S', Daniel Muñoz S², Leonor Moyano S3.
}

\begin{abstract}
RESUMEN
Introducción: La otoesclerosis es una enfermedad que produce hipoacusia de conducción que histopatológicamente corresponde a un proceso focal de remodelación ósea denominado otoespongiosis. Varios estudios describen el inicio y las características de este proceso, pero no existen trabajos que correlacionen el grado de afección histológica con el nivel de deterioro auditivo.

objetivo: Correlacionar observaciones anatomopatológicas con variables clínicas, intraoperatorias, audiométricas y evaluar el resultado de la estapedostomía.

Material y método: Estudio retrospectivo incluyendo los pacientes sometidos a estapedostomía entre 1995 y 2011, con estudio histopatológico de estribo y evaluación audiométrica pre y posquirúrgica. Estadísticamente, se hizo análisis univariado de variables categóricas y continuas, para luego ajustar modelo logístico para identificar predictores de éxito quirúrgico. Para la tabulación de datos, obtención de resultados y análisis se utilizó el software estadístico Stata ${ }^{\circledast} 12$ (StataCorp. 2011. College Station, TX: StataCorp LP).

Resultados: Se reclutaron 204 estapedostomías, sólo el 24,51\% tuvo estribos anormales. El aspecto macroscópico de la platina no tuvo relación con el resultado microscópico $(p=0,476)$, no se encontró asociación estadística entre umbrales audiométricos prequirúrgicos aéreos $(p=0,291)$ y óseos $(p=0,824)$ con el grado de severidad histopatológica. La mejoría vía aérea posquirúrgica $(p=0,326)$ y cierre del gap aéreoóseo $(p=0,23)$ no se correlacionó con el grado histológico.

Conclusión: El impacto primario de la estapedostomía fue significativo en el mejoramiento del nivel de audición del paciente. El estudio rutinario anatomopatológico de estribo en estapedostomía carece de valor pronóstico a diferencia de otros factores analizados en este estudio.
\end{abstract}

Palabras clave: Otoesclerosis, estapedostomía, histopatología, resultados auditivos.

\footnotetext{
Médico. Servicio de Otorrinolaringología, Hospital Clínico Universidad de Chile.

Médico. Universidad de Chile.

3 Médico. Servicio de Anatomía Patológica, Hospital Clínico Universidad de Chile..
} 


\section{ABSTRACT}

Introduction: Otosclerosis causes conductive hearing loss that histologically corresponds to a bone remodeling process called otoespongiosis. Although multiple pathological studies describing onset and the characteristics of this process, there are no articles in literature that correlate histological disease with hearing impairment and surgical outcomes.

Aim: Correlate pathological disease with clinical, intraoperative and audiometric variables and evaluate the stapedostomy outcome.

Material and method: Retrospective study including patients undergoing stapedostomy between 1995 and 2011, with histopathologic evaluation of stapes bone and pre and postoperative audiometric evaluation. Statistically, univariate analysis for categorical and continuous variables was used, then a logistic model to identify predictors of surgical success was adjusted. For data tabulation and analysis Stata ${ }^{\circledR} 2$ (StataCorp. 2011. College Station, TX: StataCorp LP) statistical software was used.

Results: 204 stapedectomies were assessed, 24.51\% with abnormal stapes bones, the gross appearance of the stage was not related to the microscopic result $(p=0.476)$, no association between pre-surgical air audiometric thresholds $(p=0.291)$ and bone ( $p$ $=0.824$ ) with histopathologic severity was found. The improved postoperative airway threshold $(p=0.326)$ and air-bone gap closure $(p=0.23)$ did not correlate with histology.

Conclusion: Stapedostomy primary outcome, ie, improvement in hearing thresholds, as well as the air-bone gap, were significant. Routine pathological stapes bone study had no prognostic value in contrast to other factors analyzed in this study.

Key words: Otosclerosis, stapedostomy, histopathology, audiometric outcome.

\section{INTRODUCCIÓN}

La otoesclerosis es una enfermedad localizada y primaria de la cápsula ótica y los huesecillos del oído. Se caracteriza por una remoción anormal de hueso maduro de la cápsula ótica mediante osteoclastos y su reemplazo por tejido óseo de gran espesor, celularidad y vascularización ${ }^{1}$. Este proceso (otoespongiosis), tiene predilección por la ventana oval, donde el compromiso de la platina del estribo resulta en su fijación y la consecuente hipoacusia de conducción².

La verdadera prevalencia de esta enfermedad es desconocida. Algunos reportes estiman la enfermedad clínica entre $0,5 \%$ y $1 \%$. Sin embargo, la incidencia de la enfermedad subclínica en estudios histológicos de hueso temporal puede llegar al $13 \%{ }^{3}$.

En términos histopatológicos, la ubicación más común del foco otoesclerótico es la región de la cápsula ótica anterior a la platina del estribo (región de la fissula ante fenestram ${ }^{4}$.
Previamente, hemos publicado los resultados preliminares de los aspectos anatomopatológicos de la otoesclerosis, donde se sugirió una clasificación de acuerdo a los hallazgos de tal estudio ${ }^{5}$. Esta clasificación será usada en el presente artículo (Tabla 1, Figuras 1-5).

La estapedostomía es una opción de tratamiento de la pérdida auditiva que resulta de la otoesclerosis y se ha probado como una intervención efectiva y segura $^{2}$. Habitualmente se ha considerado como medida primaria de éxito quirúrgico al cierre del gap óseo-aéreo $(\leq 10 \mathrm{~dB})$ en la literatura ${ }^{6-8}$, así como también un mejoramiento posoperatorio en la conducción aérea ( 20 dB o menos), ya que ésta refleja el grado de éxito en la restauración de la función de transmisión del sonido del oído medio?.

Si bien las características histológicas de esta enfermedad están bien documentadas, no existe en la literatura prácticamente ningún estudio que correlacione el grado de afectación histológica en otoesclerosis con el nivel de deterioro auditivo y resultados quirúrgicos en estos pacientes. 
Tabla 1. Clasificación anatomopatológica de los estribos en pacientes con otoesclerosis

\begin{tabular}{|ll|}
\hline Grado & Descripción \\
\hline 0 & Hueso o tendón normal. \\
1 & Calcificación ósea aumentada o nodularidad en el tendón. \\
2 & Aposición ósea y remodelación leve. \\
3 & Aposición ósea y remodelación moderada. \\
4 & Aposición ósea y remodelación severa. \\
\hline
\end{tabular}

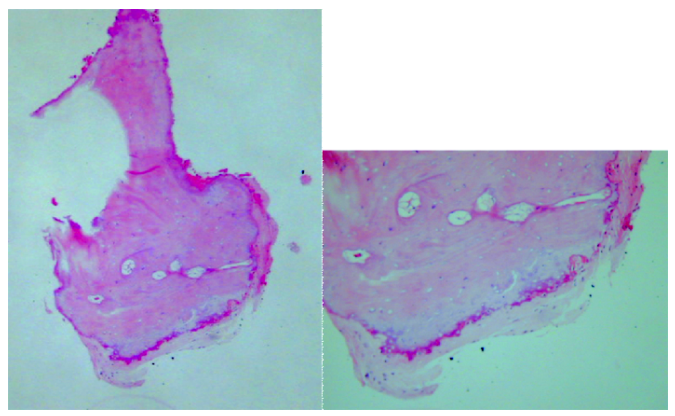

Figura 1. Grado 0. Hueso o tendón normal.

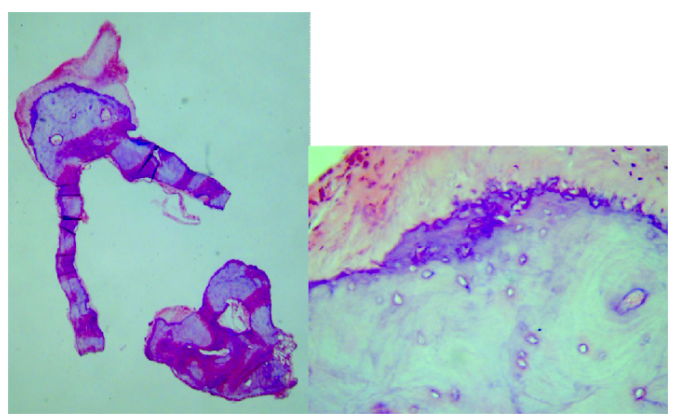

Figura 3. Grado 2. Aposición ósea y remodelación leve.

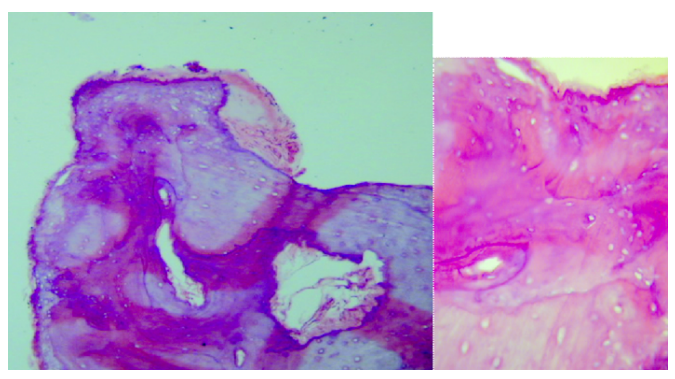

Figura 5. Grado 4. Aposición ósea y remodelación severa.

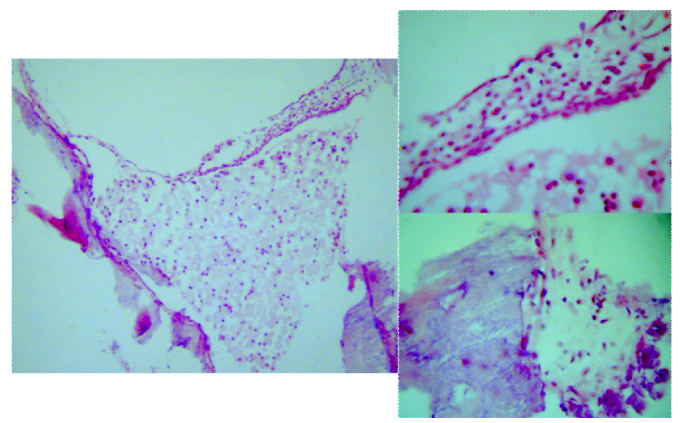

Figura 2. Grado 1. Calcificación ósea aumentada o nodularidad en el tendón.

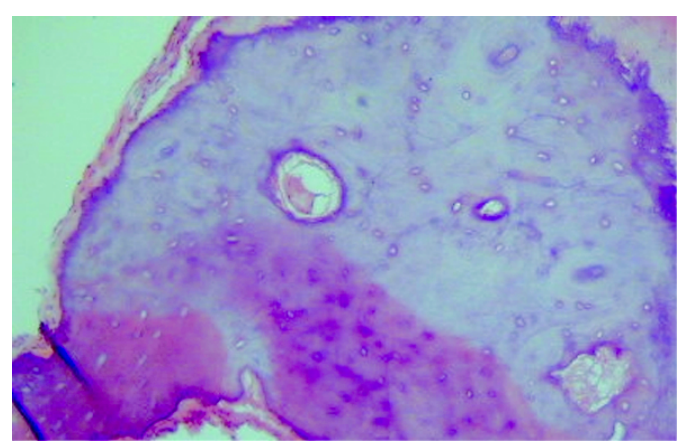

Figura 4. Grado 3. Aposición ósea y remodelación moderada.

Schuknecht, por ejemplo, no encontró correlación entre la magnitud de la pérdida auditiva y el tamaño, actividad o localización de la lesión otoesclerótica ${ }^{10}$.

\section{OBJETIVO}

El objetivo del presente estudio es verificar las características anatomopatológicas de la 
otoesclerosis, su correlación con las variables clínico-audiométricas y evaluar el resultado del tratamiento quirúrgico en nuestros pacientes.

\section{MATERIAL Y MÉTODO}

Estudio de cohorte retrospectivo donde se incluyeron los pacientes sometidos a estapedostomía primaria entre los años 1995 y 2011, que cuentan con estudio histopatológico de estribo y evaluación audiométrica pre y posquirúrgica en el Hospital Clínico de la Universidad de Chile.

Se recabaron antecedentes clínico-demográficos (edad, sexo, tiempo entre cirugía a la realización de la biopsia, bilateralidad), audiométricos tales como umbrales auditivos (promedio tonal puro) de vía ósea, aérea y gap pre y posestapedostomía y de la intervención quirúrgica (oído operado, aspecto de la platina), mediante revisión fichas clínica y del protocolo operatorio.

El procedimiento quirúrgico consistió en estapedostomía con prótesis de Schuknecht ${ }^{\circledR}$ de 4,5 $\mathrm{mm}$ de largo y $06 \mathrm{~mm}$ de diámetro, realizadas por el mismo cirujano otorrinolaringólogo y su equipo.

Todas las piezas quirúrgicas fueron enviadas al Servicio de Anatomía Patológica del Hospital Clínico de la Universidad de Chile, procesados bajo técnica estándar y analizados por el mismo patólogo. Los estribos se categorizaron según la clasificación propuesta. Tabla 1. Clasificación anatomopatológica de los estribos en pacientes con otoesclerosis.

\section{Análisis estadístico}

Para la tabulación de datos, obtención de resultados y análisis se utilizó el software estadístico Stata ${ }^{\circledR} 12$ (StataCorp. 2011. College Station, TX: StataCorp LP).

Primeramente, se evalúa la asociación entre el grado histopatológico y algunas variables clínicas tales como edad, sexo y umbrales auditivos previos a la cirugía. Para la comparación de variables categóricas se utiliza el test exacto de Fisher y $\mathrm{V}$ de Cramer para cuantificación de la asociación. Para las variables continuas se utilizó la prueba ANOVA de un factor.

Para evaluar el éxito quirúrgico se comparan los resultados audiométricos pre y posquirúrgicos, mediante el test $t$ de Student para muestras pareadas.

Además, se ajustan paralelamente 2 modelos de regresión logística multivariada con las variables clínicas y audiométricas que pudieren explicar el resultado de la cirugía, a fin de identificar los factores pronósticos de éxito, es decir, cierre del gap óseo-aéreo ( $\leq 10 \mathrm{~dB}$ ) y ganancia vía aérea (20 dB o menos) poscirugía.

El modelo de regresión logística se ajustó verificando la calidad del mismo y su bondad de ajuste mediante el área bajo la curva ROC y la prueba de Hosmer-Lemeshow, respectivamente. Como medida de asociación entre las variables pronósticas y el éxito quirúrgico se obtiene odds ratio $(\mathrm{OR})$ con su respectivo intervalo de confianza. En toda dócima de hipótesis se consideró como significativo un valor $p$ menor a 0,05 .

\section{RESULTADOS}

Se reclutaron finalmente 204 estapedostomías correspondientes a 175 pacientes entre los años 1995 y 2011, cuyas características clínico demográficas se presentan en la Tabla 2.

Tabla 2. Características generales de los pacientes en estudio

\begin{tabular}{|lc}
\hline Edad, años prom. (rango) & $44,62(21-82)$ \\
Sexo, \% & $61,07 \%$ femenino; $38,93 \%$ masculino \\
Oído operado, \% & $51,01 \%$ derecho; 48,99 izquierdo \\
Bilateralidad, \% & 62,31 \\
Tiempo biopsia, días prom. (DE) & $2(4,22)$ \\
Audiometría poscirugía, días prom. (DE) & $48,77(11,36)$
\end{tabular}


La distribución de las biopsias de estribo según la clasificación antes mencionada mostró un claro predominio del grado 0 (estribos normales) con el $75,49 \%$ de los casos. El detalle se presenta en la Figura 6.

En cuanto a esta distribución no se encontró asociación estadísticamente significativa entre ésta y variables como sexo $(p=0,352)$, edad $(p=0,237)$ y resultados audiométricos prequirúrgicos tales como umbrales de vía aérea $(p=0,291)$ y de vía ósea $(p=0,824)$. Es decir, no por mayor severidad histopatológica necesariamente hay mayor deterioro auditivo. Algo similar ocurre al considerar el grado histopatológico como predictor de mejoría de la vía aérea $(p=0,211)$ y cierre del gap óseoaéreo $(p=0,093)$, pues no se encontró asociación entre las variables.

El impacto primario de la estapedostomía fue significativo en el mejoramiento de los umbrales auditivos de vía aérea, así como también el gap óseoaéreo (Tabla 3). En términos porcentuales, tenemos que el $82,35 \%$ ( $n=168)$ de los oídos intervenidos presentó mejoría de la vía ósea y el $65,19 \%(n=133)$ presentó cierre del gap óseo-aéreo.

La presentación del aspecto macroscópico de la platina no tuvo relación con el resultado microscópico (Figura 7). Si bien existe cierta agregación entre el aspecto azul de la platina del estribo y el grado 0 histopatológico (normal), no existe asociación estadísticamente significativa (V de Cramer $=0,18 ; p=0,476$ ).

Con respecto a la evaluación del éxito quirúrgico y su asociación con las variables pronósticas de éste, tenemos que la mejoría en vía aérea $(20 \mathrm{~dB} 0$ menos) se correlaciona bien con variables edad $(0 R=0,94[0,89-0,99] ; p=0,035)$ y umbral aéreo prequirúrgico ( $O R=0,85[0,77-0,91] ; p=0,001)$. No ocurre así para las variables sexo $(O R=1,45$
$[0,49-4,28] ; p=0,496)$, grado histológico (OR $=0,74 \quad[0,41-1,33] ; \quad p=0,326)$, umbral óseo prequirúrgico ( $O R=0,99$ [0,99-1,02]; $p=0,43)$, gap óseo-aéreo prequirúrgico $(\mathrm{OR}=1,05$ [0,96-1,14]; $p$ $=0,23)$. El modelo así ajustado obtuvo un área bajo la curva ROC de 0,74 , es decir, existió $74 \%$ de predicción correcta y una bondad de ajuste buena ( $R V=16,15 ; p=0,01$ y Hosmer-Lemeshow con chi ${ }^{2}$ $=92,57 ; p=0,1785$ ).

En el caso del éxito quirúrgico en términos del cierre del gap óseo-aéreo, tenemos que bajo el modelo ajustado, dicho éxito se correlacionó positivamente sólo con el umbral de vía ósea prequirúrgico $(0 R=1,06[1,03-1,13] ; p=0,039)$ y el gap prequirúrgico $(O R=0,63[0,61-0,77] ; p$ $=0,0002)$. El modelo así ajustado obtuvo un área bajo la curva ROC de 0,7021, es decir, existió $70,21 \%$ de predicción correcta y una bondad de ajuste buena (RV $=13,25 ; p=0,031$ y HosmerLemeshow con chi $\left.^{2}=105,94 ; p=0,1519\right)$.

\section{DISCUSIÓN}

Nuestro estudio muestra una clara predominancia por la presencia de otosclerosis en el sexo femenino y una edad al momento de la cirugía cercana a la $5^{\text {a }}$ década de vida. Este hecho guarda relación con otras casuísticas en términos epidemiológicos ${ }^{7,11}$. Se constató también que el oído más intervenido fue el derecho junto con un alto porcentaje de bilateralidad, algo que se vio en una serie anterior ${ }^{5}$.

La distribución de los hallazgos anatomopatológicos mostró preponderancia de estribos normales (Grado 0) en el 75,49\% de las muestras analizadas. Esto se condice con la descripción de la literatura que sostiene que sería raro encontrar focos de otoespongiosis en los huesecillos ${ }^{12}$.

Tabla 3. Resultados audiométricos pre y posestapedostomía en pacientes con otoesclerosis

\begin{tabular}{|lllll|}
\hline Variable & $\begin{array}{l}\text { Prequirúrgico } \\
\text { (IC 95\%) dB }\end{array}$ & $\begin{array}{l}\text { Posquirúrgico } \\
\text { (IC 95\%) dB }\end{array}$ & $\begin{array}{l}\text { Cambio } \\
\text { (IC 95\%) dB }\end{array}$ & P Value \\
\hline Vía ósea & $35,46(32,92-38,01)$ & $18,56(16,59-20,52)$ & $16,9(14,98-18,83)$ & $<0,0001$ \\
Vía aérea & $59,36(56,4-62,32)$ & $26,58(23,59-29,56)$ & $32,78(30,13-35,43)$ & $<0,0001$ \\
Gap óseo-aéreo & $34,27(32,13-36,41)$ & $9,12(7,31-10,94)$ & $25,14(22,51-27,77)$ & $<0,0001$ \\
\hline
\end{tabular}




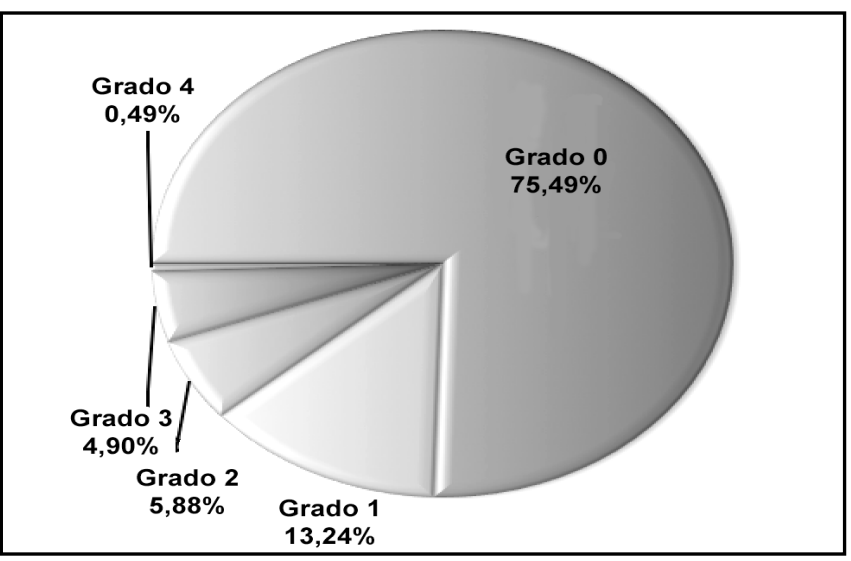

Figura 6. Distribución según la clasificación anatomopatológica propuesta, de los estribos en pacientes con otoesclerosis.

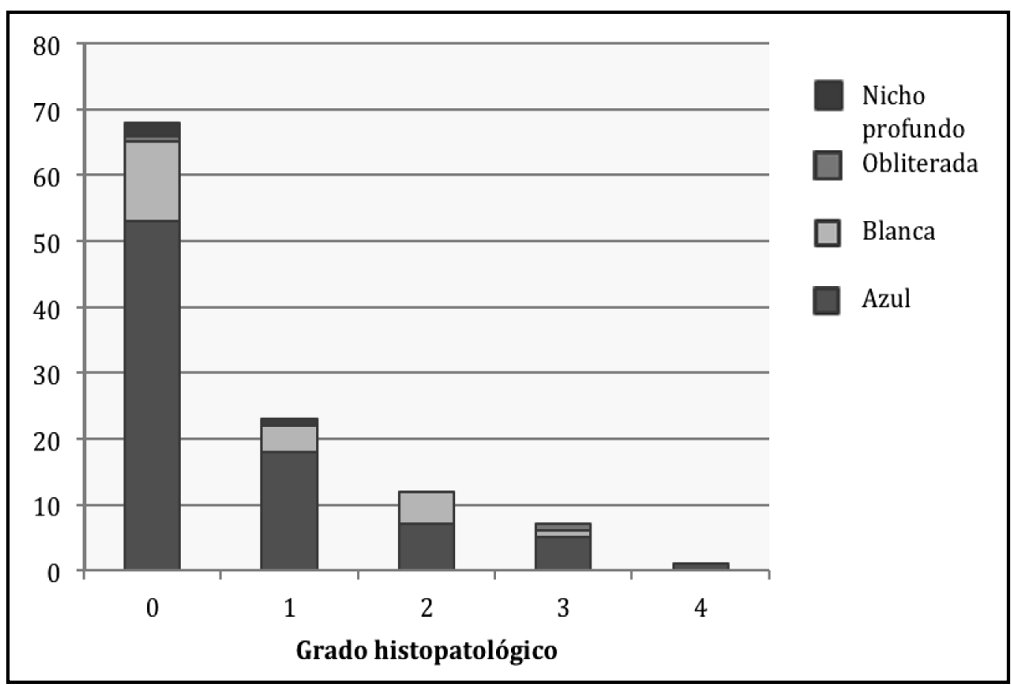

Figura 7. Distribución de frecuencia según grado histopatológico y aspecto macroscópico de la platina del estribo.

La presentación macroscópica de la platina tampoco se relaciona con la evaluación anatomopatológica, ya que la distribución de la platina azul, blanca u obliterada podría considerarse similar en los distintos grados histológicos. Este podría explicarse porque el foco de otoespongiosis sería distinto al de la fissula antefenestray, al desarrollarse la enfermedad, afectaría primero los huesecillos y muy tardíamente la platina, o al contrario el foco de otoespongiosis estaría en la fissula antefenestra y se propagaría rápidamente por la platina alcanzando la supraestructura del estribo $0^{5,13,14}$.
Los resultados auditivos de la estapedostomía en nuestra serie se correlacionan con lo reportado en la literatura, existiendo mejoría significativa en la conducción de sonido por vía aérea $(82,35 \%$ de los oídos intervenidos) y reducción del gap óseoaéreo posquirúrgico $(65,19 \%$ de los oídos intervenidos). Sólo por mencionar algunos, en los estudios de Bitterman y $\operatorname{cols}^{15}(80,1 \%$ de mejoría en vía aérea y $62,1 \%$ de cierre de gap) y el de Van Rompaey y $\operatorname{cols}^{16}(92,6 \%$ de mejoría en vía aérea y $63,6 \%$ de cierre en gap), se aprecian similares resultados quirúrgicos a los de nuestra serie, aportando más evidencia a favor de la estapedostomía 
como un procedimiento efectivo y seguro en el tratamiento de la otoesclerosis.

Como se mencionó anteriormente, con respecto a la evaluación del éxito quirúrgico y su asociación con las variables pronósticas de éste, tenemos que la mejoría en vía aérea se correlacionó con la edad y el umbral aéreo prequirúrgico. Esto significa que en términos predictivos, una edad mayor al momento de la cirugía se asocia con un menor éxito quirúrgico en cuanto al mejoramiento de la vía aérea. Lo mismo ocurre para el umbral aéreo prequirúrgico, es decir, a mayor deterioro de éste menor probabilidad de obtener 20 dB o menos de vía aérea en el posoperatorio. En el caso del cierre del gap óseo-aéreo se tuvo que éste correlacionó con el gap prequirúrgico y el umbral de vía ósea prequirúrgica. Esto significa que el cierre del gap óseo-aéreo ocurre con mayor probabilidad si al momento de la cirugía existe un mayor deterioro de la transmisión sonora por vía ósea, lo que es lógico pues es más sencillo acercar la vía aérea a una ósea más deteriorada. En el caso del cierre del gap y el gap prequirúrgico, existe una asociación inversa en el sentido de que un gap preoperatorio mayor predice un menor cierre en el posoperatorio, algo también lógico pues se asume una lesión de huesecillos mayor. Se requerirá en futuras investigaciones, correlacionar el éxito quirúrgico con otras variables tales como dehiscencia del facial, platinas flotantes $u$ otras observaciones intraoperatorias.

Finalmente, en cuanto a la relación entre el daño histológico con el daño auditivo previo y la predicción de su mejoría con la intervención quirúrgica, no se encontró correlación estadística entre las variables. Es decir, no por mayor severidad histopatológica necesariamente hay mayor severidad del deterioro auditivo en el estado prequirúrgico y menor mejoría del estado auditivo posestapedostomía.

\section{CONCLUSIONES}

El tratamiento quirúrgico, específicamente la estapedostomía, es claramente beneficiosa en términos de mejorar la audición de los pacientes. Obviamente, esta decisión debe ser tomada por el paciente, bajo consentimiento informado. El estudio anatomopatológico del estribo en otoesclerosis, carece de valor clínico y debe reservarse sólo con fines investigativos. Es por ello, que la otoesclerosis debe seguir considerándose una enfermedad eminentemente de diagnóstico clínico y audiológico.

\section{BIBLIOGRAFÍA}

1. Lee KJ, ed. Essential Otolaryngology, Head and Neck Surgery. $9^{\text {th }}$ ed. Mc Graw Hill, 2008.

2. Karosi T, Sziklal I. Etiopathogenesis of otosclerosis. Eur Arch Otorhinolaryngol 2010; 267(9): 1337-49.

3. Lalwani A, ed. Current Diagnosis and Treatment. Otolaryngology Head and Neck Surgery. $2^{\text {nd }}$ ed. Mc Graw Hill, 2008.

4. Linthicum FH JR. Histopathology of otosclerosis. Otolaryngol Clin North Am 1993; 26(3): 335-52.

5. Stott C, Arteaga P, Moyano L. ¿Qué sabemos de otoesclerosis? Aspectos anatomopatológicos. Stott C, Arteaga P, Moyano L. Rev Otorrinolaringol Cir Cabeza Cuello 2005; 65: 179-186.

6. KisileVSKKY VE, Dutt SN, BaILIE NA, Halik JJ. Hearing results of 1145 stapedotomies evaluated with Amsterdam hearing evaluation plots. J Laryngol Otol 2009; 123(7): 730-6.

7. Vincent R, Sperling NM, OAtes J, Jindal M. Surgical findings and long-term hearing results in 3,050 stapedotomies for primary otosclerosis: a prospective study with the otology-neurotology database. Otol Neurotol 2006; 27(8)(suppl 2): S25-S47.

8. de Bruijn AJ, Tange RA, Dreschler WA. Efficacy of evaluation of audiometric results after stapes surgery in otosclerosis. Otolaryngol Head Neck Surg 2001; 124(1): 76-83.

9. de Bruijn AJ, Tange RA, Dreschler WA. Efficacy of evaluation of audiometric results after stapes surgery in otosclerosis, II. Otolaryngol Head Neck Surg 2001; 124(1): 84-9.

10. SCHUKNeCht HF, Barber W. Histologic variants in otosclerosis. Laryngoscope 1985; 95(11): 130717.

11. Merán Gill Jl, Masgoret Palau E, Avilés Jurado FJ, Domènech Vadillo E, Flores Martín JC, Figuerola Massana E. Stapedotomy outcomes in the treatment of otosclerosis: our experience. Acta Otorrinolaringol Esp 2008; 59(9): 448-54. 
12. Declau F, van Spaendonck M, Timmermans JP, Michaels L, Liang J, Qiu JP, van de Heyning P. Prevalence of histologic otosclerosis: an unbiased temporal bone study in Caucasians. Adv Otorhinolaryngol 2007; 65: 6-16.

13. Michaels L, Soucek S. Origin and growth of otosclerosis. Acta Otolaryngol 2011; 131(5): 460-8.

14. Cureoglu S, Schachern Pa, Ferlito A, Rinaldo A, Tsuprun V, Paparella MM. Otosclerosis: etiopathogenesis and histopathology. Am J Otolaryngol 2006; 27(5): 334-40.
15. Bittermann AJ, Rovers MM, Tange RA, Vincent R, Dreschler WA, Grolman W. Primary stapes surgery in patients with otosclerosis: prediction of postoperative outcome. Arch Otolaryngol Head Neck Surg 2011; 137(8): 780-4.

16. Van Rompaey V, Yung M, Claes J, Häusler R, Martin C, Somers T, Offeciers E, Pytel J, Skladzien J, de HeYning PV. Prospective effectiveness of stapes surgery for otosclerosis in a multicenter audit setting: feasibility of the Common Otology Database as a benchmark database. Otol Neurotol 2009; 30(8): 1101-10.

Dirección: Dr. Alejandro Ojeda S.

Hospital Clínico Universidad de Chile. Santos Dumont 999, Independencia. Santiago.

E mail: arojedas@gmail.com 